\title{
Photocatalytic growth of dendritic silver nanostructures as SERS substrates $\dagger \ddagger$
}

\author{
Katrien G. M. Laurier, ${ }^{a}$ Michel Poets, ${ }^{b}$ Frederik Vermoortele, ${ }^{b}$ Gert De Cremer, ${ }^{b}$ \\ Johan A. Martens, ${ }^{b}$ Hiroshi Uji-i, ${ }^{a}$ Dirk E. De Vos, ${ }^{b}$ Johan Hofkens ${ }^{a}$ and \\ Maarten B. J. Roeffaers $* a b$
}

Received 31st July 2011, Accepted 27th September 2011 DOI: $10.1039 / \mathrm{c} 1 \mathrm{cc} 14727 \mathrm{~g}$

\begin{abstract}
We report a one-step photocatalytic synthesis method of dendritic silver nanostructures. These self-organised structures show an excellent Raman enhancement enabling the detection of analytes from dilute solutions by surface-enhanced Raman spectroscopy.
\end{abstract}

The properties of nanostructured metals change drastically with small variations in size, morphology, crystallinity, organization and composition. Due to this strong variability in properties, metal nanoparticles have attracted a lot of interest over the last decade. Not only have these studies focused on the controlled synthesis and growth of metallic nanostructures; many reports also explored the applications of such metallic nanoparticles in optics, electronics, as catalysts or as sensors. Many recipes are now known for the generation of nanostructured metals. One of the most intensely investigated fields is the application of metallic nanostructures as substrates for Surface Enhanced Raman Scattering (SERS). ${ }^{1,2}$ Here, the metallic surface serves to locally enhance the electromagnetic fields via its ability to sustain surface plasmons. ${ }^{1,3}$ As a result the weak Raman scattering process is enhanced by several orders of magnitude, making it possible to detect minute amounts of analytes by recording vibrational fingerprints even at the single molecule level. ${ }^{4}$ The excitation of surface plasmons by photons strongly depends on the used metal, with silver structures being the material of choice for visible light stimulation. ${ }^{3}$ The size and the shape of the nanoparticles also play an important role in the enhancement as SERS hotspots occur at edges, surface steps and nanoscale features. ${ }^{2}$ In this context hierarchical nanostructures such as dendritic silver are an interesting group of metal structures since they can grow to microscale assemblies via self-organization, while sustaining a

\footnotetext{
${ }^{a}$ Department of Chemistry, Katholieke Universiteit Leuven, Celestijnenlaan 200F, B-3001, Heverlee, Belgium

${ }^{b}$ Department of Microbial and Molecular Systems,

Katholieke Universiteit Leuven, Kasteelpark Arenberg 23, B-3001,

Heverlee,Belgium.E-mail: maarten.roeffaers@biw.kuleuven.be;

Fax: + 3216 327990; Tel: + 3216327920

$\dagger$ This article is part of the ChemComm 'Emerging Investigators 2012' themed issue.

$\ddagger$ Electronic supplementary information (ESI) available: $\mathrm{ZnO}$ synthesis, photocatalytic experiment; SEM measurements, fluorescence and Raman microscopy; photocatalytic water oxidation. See DOI: $10.1039 / \mathrm{c} 1 \mathrm{cc} 14727 \mathrm{~g}$
}

large concentration of nanoscale surface features. ${ }^{5-7}$ Furthermore, these hierarchically organized structures exhibit a large surface area which enhances the interaction with the analytes in sensing applications. ${ }^{6}$

Various shape-selective synthesis methods have been developed, generating hierarchical silver nanostructures. Many of these methods employ organic reagents that act as an electron source, surfactant and/or a capping agent. ${ }^{7}$ These organics often remain strongly bound to the metal surface complicating the purification and limiting their use as easy-to-fabricate SERS substrates.

Herein, we report on a simple, organics-free method to prepare hierarchical silver nanostructures via a room temperature photocatalytic reduction of silver ions directly from an aqueous solution on zinc oxide $(\mathrm{ZnO})$ crystals. This additive-free method works directly on top of optically transparent cover slides and does not rely on metallic support or substrate structures. ${ }^{6}$ Hence this preparation method uniquely enables direct application of dendritic silver structures as SERS substrates for sensing analytes. Raman mapping of the micrometre-sized dendritic silver structures shows excellent SERS enhancement throughout the whole dendritic structure which is promising for use as sensor.

When a sample of white zinc oxide powder, deposited on a glass cover slide and submerged in a $1 \mathrm{mM}$ silver nitrate solution, is illuminated with UV light (315-400 nm) inside a photoreactor, it becomes slightly grey. This darkening of the $\mathrm{ZnO}$ particles, related to the photocatalytic reduction of silver ions, increases with illumination time. The UV illumination of $\mathrm{ZnO}$ crystals induces the formation of electron/hole pairs which after diffusion to the surface can perform redox reactions, e.g. the reduction of silver ions by photoelectrons. ${ }^{8}$ This photocatalytic silver reduction by $\mathrm{ZnO}$ crystals was visualized with an optical microscope. (Detailed information on the synthesis of the $\mathrm{ZnO}$ crystals, the photocatalytic experiments and the confocal microscope is listed in the ESI. $\ddagger$ ) The pure, as-synthesized $\mathrm{ZnO}$ crystals appear as large hexagonal prisms in the optical transmission image (Fig. 1a). The visually observed darkening of the $\mathrm{ZnO}$ powder upon exposure to $\mathrm{Ag}^{+}$under UV stimulation translates into a clear darkening of the individual $\mathrm{ZnO}$ crystals in the optical transmission images (Fig. 1b). This darkening of the crystals however does not occur homogeneously, isolated silver particles appear at the 


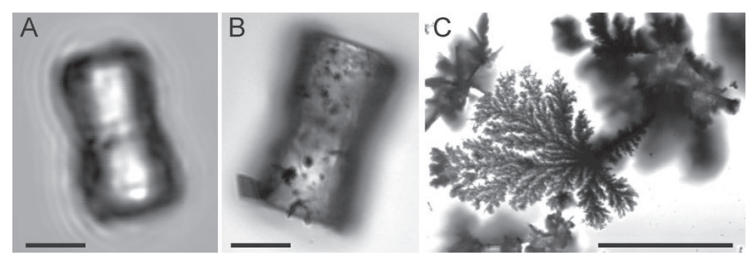

Fig. 1 Photocatalytic generation of dendritic silver structures from an aqueous $\mathrm{AgNO}_{3}$ solution $(1 \mathrm{mM})$ inside a photoreactor: (A) pure $\mathrm{ZnO}$ crystal, scale bar $=10 \mu \mathrm{m}$; (B) heterogeneous deposition of silver nanoparticles, scale bar $=10 \mu \mathrm{m}$; (C) growth of dendritic silver nanostructures, scale bar $=50 \mu \mathrm{m}$.

$\mathrm{ZnO}$ crystals outer surface. Hence, instead of forming a homogeneous silver coating the photocatalytic silver reduction occurs more rapidly in certain zones. ${ }^{9}$

This heterogeneous silver metal deposition becomes even more pronounced upon extended UV stimulation in the presence of silver ions. The optical images of these samples show the development of a limited number of dendritic metal structures for every $\mathrm{ZnO}$ crystal. The trunk of these highly branched structures is attached to the surface of the zinc oxide crystal. The size of these structures increases with illumination time, extending up to $100 \mu \mathrm{m}$ in length after long UV stimulation of the $\mathrm{ZnO}$ core. Fig. 1c shows such a remarkably large, individual silver dendrite formed on a $\mathrm{ZnO}$ photocatalyst. When similar experiments were performed with $10 \times$ lower or $100 \times$ higher $\mathrm{Ag}^{+}$concentration the formation of such large structures was not observed. A more detailed view on the dendritic silver structures comes from scanning electron micrographs (ESI $\ddagger$ ).

Next, the photocatalytic growth of metallic silver nanostructures on the $\mathrm{ZnO}$ crystals was visualized in situ directly on top of the inverted confocal fluorescence microscope (ESI $\ddagger$ ). The zinc oxide crystals are deposited on a glass cover slide and subsequently immersed in an aqueous solution containing $1 \mathrm{mM}$ silver nitrate. ${ }^{10}$ The $\mathrm{ZnO}$ crystals are clearly visible as large hexagonal prisms in the optical transmission image (Fig. 2a), with only faint green-yellow luminescence being detected in the confocal fluorescence image (Fig. 2b). ${ }^{11}$ Both images were obtained by laser scanning the sample with a green laser (ESI $\ddagger$ ). Upon simultaneous wide-field UV stimulation $(<400 \mathrm{~nm})$ the luminescence intensity increases because of the
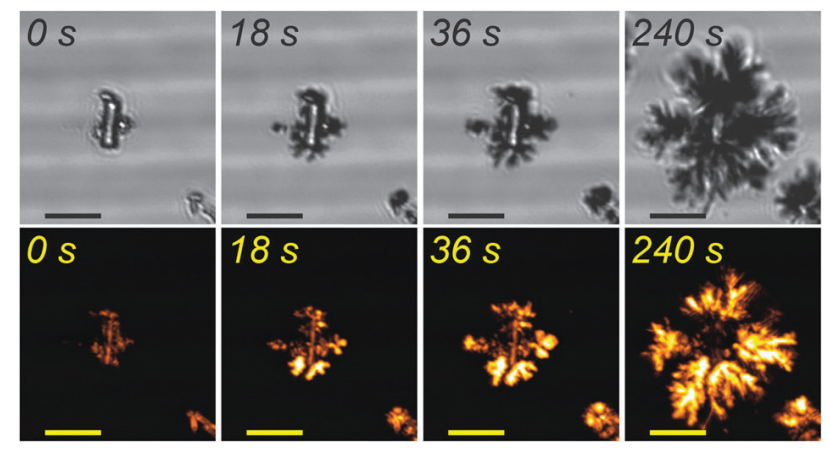

Fig. 2 In situ observation of the photocatalytic generation of dendritic silver structures at $0 \mathrm{~s}, 18 \mathrm{~s}, 36 \mathrm{~s}$, and $240 \mathrm{~s}$ after starting the UV illumination: (A) optical transmission images recorded, (B) corresponding luminescence images (excitation $=543 \mathrm{~nm}$ and luminescence $=550-650 \mathrm{~nm})$. Scale bar $=10 \mu \mathrm{m}$. strongly enhanced $\mathrm{ZnO}$ excitation. Shortly after switching on the wide-field UV illumination, the optical transmission image reveals several isolated silver nanoparticles, appearing as dark spots, at the $\mathrm{ZnO}$ surface similar as in the samples prepared in the photoreactor. Remarkably, a strong luminescence signal is detected at these silver nanoparticles in the fluorescence channel (550-650 nm). Further UV stimulation results in the growth of well separated dendritic silver structures. This anisotropic growth pattern is generally associated with a non-equilibrium, diffusion-limited aggregation model. ${ }^{12}$ The initially formed reduced silver nanoparticles act as an electron sink to which photoelectrons, formed after continued UV stimulation of the $\mathrm{ZnO}$ crystal, can quickly migrate. ${ }^{13}$ These electrons are subsequently used for further silver ion reduction.

Whereas development of large dendritic nanostructures was mainly observed in $1 \mathrm{mM}$ silver nitrate solutions, at elevated concentrations next to the photocatalytic silver reduction, also the second photocatalytic half reaction, i.e. water oxidation, was observed as the formation of oxygen gas bubbles (ESI $\ddagger$ ).

Important for the generation of effective SERS substrates with high enhancement factors is the presence of a large concentration of (sub-)nanometre-sized features. In this case, the generated hierarchical silver microstructures should contain SERS hotspots throughout the dendritic structure. Evidence for the presence of such (sub-)nanometre-sized features and nanoclusters comes from the luminescence that these dendritic structures generate (Fig. 2b). This indicates that the dendritic silver is not one continuous metal structure, which should not luminesce, but growth occurs in a rather heterogeneous fashion in the presence of multiple silver nano-features/clusters. It is well-documented that such clusters can exhibit very bright luminescence. ${ }^{14}$ Furthermore, when zooming in on the micrometre-sized dendritic silver the structures show blinking luminescent dots of diffraction-limited size indicating the presence of bright emissive silver clusters that behave as individual particles (ESI $\ddagger$ ). This bright luminescence with green laser excitation however does not limit the use of these structures as SERS substrates, vide infra.

Fig. 3a shows the surface-enhanced Raman spectrum (633 nm excitation, outside the excitation spectrum of the aforementioned emissive clusters) of an ethanolic solution of $100 \mathrm{nM}$ and $1 \mu \mathrm{M}$ 4-MOTP (4-methoxythiophenol) measured at the dendritic silver compared to that of $100 \mu \mathrm{M}$ 4-MOTP measured under the same experimental conditions but in the absence of a SERS substrate. Whereas the former spectra clearly show the vibrational fingerprint of 4-MOTP, ${ }^{15}$ the latter spectrum only contains minor peaks originating from the solvent, ethanol, with no peaks of 4-MOTP being resolved. Raman mapping experiments were conducted to assess whether the enhancement is coming from some selected spots or from the whole micrometre-sized dendritic silver structure. Fig. 3b shows an optical transmission image of the dendritic silver structure with the corresponding photoluminescence image (Fig. 3c, emission 650-750 nm) and Raman map (Fig. 3d, $1060-1120 \mathrm{~cm}^{-1}$ ) recorded in the presence of $100 \mu \mathrm{M} 4$-MOTP in ethanol. To reconstruct the luminescence and Raman image, individual SERS spectra were recorded using a piezoelectric scanning stage and probing an area of $30 \times 30 \mu \mathrm{m}^{2}$ with $32 \times$ 32 pixels (ESI $\ddagger$ ). At every point the Raman spectrum was 
A
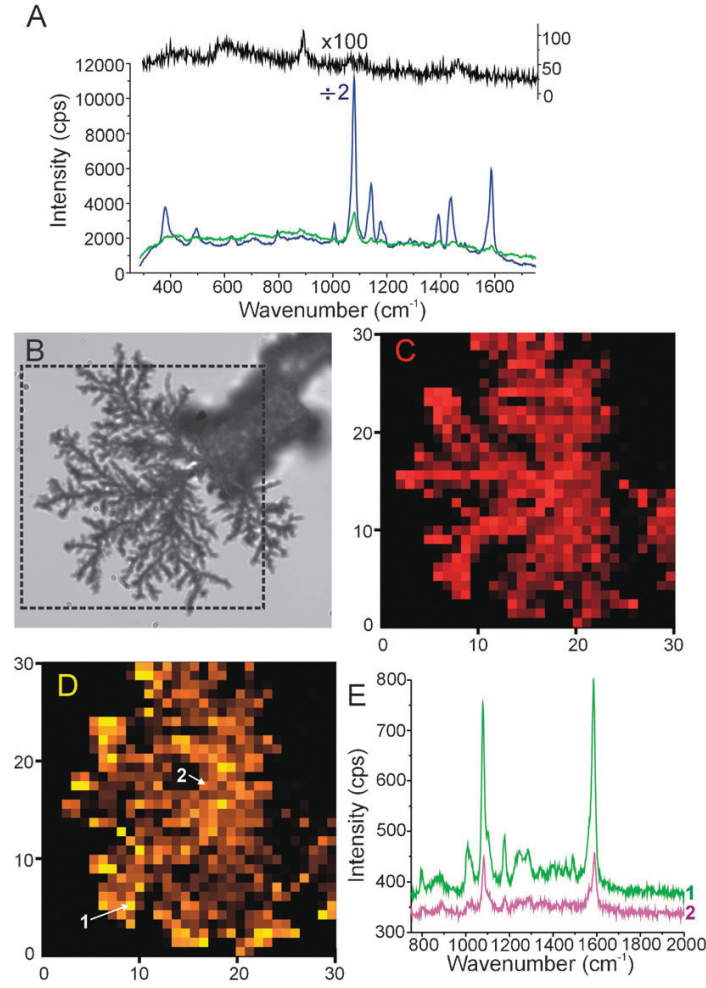

Fig. 3 Dendritic silver nanostructures as SERS substrates: (A) comparison of the Raman spectrum of an ethanolic solution containing 4-MOTP recorded in the presence (blue, green) and absence (black) of the SERS substrate; concentration of 4-MOTP is $100 \mathrm{nM}$ (green), $1 \mu \mathrm{M}$ (blue) and $100 \mu \mathrm{M}$ (black). (B)-(D) Micrographs of dendritic silver structures submerged in an ethanolic 4-MOTP solution: (B) optical transmission image, (C) photoluminescence image (650-750 nm) and (D) Raman map $\left(1060-1120 \mathrm{~cm}^{-1}\right)$. (E) Two representative SERS spectra extracted from the Raman map (D) at positions indicated with 1 and 2.

recorded during 1 second; two representative spectra are shown in Fig 3e. As usual, these SERS spectra contain a broad continuum emission, which is generally referred to as background. ${ }^{15}$ The integrated background signal between 500 and $2500 \mathrm{~cm}^{-1}$, corresponding to an emission in the range of approx. $650 \mathrm{~nm}$ to $750 \mathrm{~nm}$, was used to plot the luminescence image (ESI $\ddagger$ ). The whole dendritic structure shows a constant background emission (the origin of this background is still lively debated in the literature $)^{15}$ on top of which intense Raman peaks are present. The pronounced Raman peak at $1075 \mathrm{~cm}^{-1}$ stemming from the $\mathrm{C}-\mathrm{S}$ stretching vibration of 4-MOTP molecules was integrated and used to reconstruct the Raman map (Fig. 3d). Also here it is obvious that the whole dendritic structure shows excellent Raman enhancement and that this enhancement is reasonably homogeneously. This homogeneous Raman enhancement over large areas is very important for sensing applications. These results can be easily reproduced for most dendrites formed via this method. Upon prolonged measurements at one position no changes in the SERS spectra were observed. Whereas the generation of large area SERS sensors normally requires strictly controlled deposition of metallic nanoparticles over several length scales to yield a homogeneous distribution of hotspots, this photocatalytic growth method yields dendritic nanostructures with similar properties in one step. Note that outside the dendritic structure, i.e. in solution or at the $\mathrm{ZnO}$ crystal, no Raman signal or luminescence are detected.

In summary, large dendritic silver nanostructures were grown photocatalytically on $\mathrm{ZnO}$ crystals directly from an additive-free silver nitrate solution. These dendritic structures are excellent SERS substrates since they yield strong Raman enhancements over large areas and they can be grown on top of optically transparent substrates.

The authors thank the 'Fonds voor Wetenschappelijk Onderzoek' (Grants G.0402.09, G0413.10, G0697.11, G0197.11 and postdoctoral fellowships for GDC and MBJR), the K.U.Leuven Research Fund (GOA 2011/03, CREA2009), the Flemish government (Methusalem funding - CASAS METH/08/04) and the Federal Science Policy of Belgium (IAP-VI/27) for financial support. H.U thanks the Japanese Society for the promotion of Science and Technology for a PRESTO grant. This article is part of the Chem. Comm. 'Emerging Investigators 2012' themed issue.

\section{Notes and references}

1 R. J. C. Brown and M. J. T. Milton, J. Raman Spectrosc., 2008, 39, 1313-1326.

2 N. J. Halas, S. Lal and W. S. Chang, et al., Chem. Rev., 2011, 111, 3913-3961; M. L. Tran, S. P. Centeno and J. A. Hutchison, et al., J. Am. Chem. Soc., 2008, 130, 17240-17241; J. A. Hutchison, S. P. Centeno and H. Odaka, et al., Nano Lett., 2009, 9, 995-1001.

3 K. A. Willets and R. P. Van Duyne, Annu. Rev. Phys. Chem., 2007, 58, 267-297.

4 S. M. Nie and S. R. Emery, Science, 1997, 275, 1102-1106; S. Habuchi, M. Cotlet and R. Gronheid, et al., J. Am. Chem. Soc., 2003, 125, 8446-8447; K. Kneipp, Y. Wang and H. Kneipp, et al., Phys. Rev. Lett., 1997, 78, 1667-1670.

5 L. L. He, et al., J. Raman Spectrosc., 2010, 41, 739-744; Y. Y. Xia and J. M. Wang, Mater. Chem. Phys., 2011, 125, 267-270; W. C. Ye, C. M. Shen and J. F. Tian, et al., Solid State Sci., 2009, 11, 1088-1093.

6 A. Gutes, C. Carraro and R. Maboudian, J. Am. Chem. Soc., 2010, 132, 1476-1477; X. C. X. Chen, C. H. Cui and Z. Guo, et al., Small, 2011, 7, 858-863; Z. Zheng, S. C. Tang and S. Vongehr, et al., Mater. Chem. Phys., 2011, 129, 594-598.

7 X. Q. Wang, H. Itoh and K. Naka, et al., Langmuir, 2003, 19, 6242-6246; M. H. Rashid and T. K. Mandal, J. Phys. Chem. C, 2007, 111, 16750-16760; V. V. Agrawal, G. U. Kulkarni and C. N. R. Rao, J. Colloid Interface Sci., 2008, 318, 501-506; X. W. Zheng, L. Y. Zhu and A. H. Yan, et al., J. Colloid Interface Sci., 2003, 268, 357-361; P. Gao, M. Zhang and H. Hou, et al., Mater. Res. Bull., 2008, 43, 531-538; J. P. Xiao, Y. Xie and R. Tang, et al., Adv. Mater., 2001, 13, 1887-1891.

8 R. Ameloot, et al., Adv. Mater., 2011, 23, 1788-1791.

9 M. B. J. Roeffaers, B. F. Sels and H. Uji-i, et al., Nature, 2006, 439, 572-575; G. De Cremer, B. F. Sels and D. E. De Vos, et al., Chem. Soc. Rev., 2010, 39, 4703-4717.

10 M. B. J. Roeffaers, et al., ChemPhysChem, 2005, 6, 2295-2299.

11 R. Georgekutty, M. K. Seery and S. C. Pillai, J. Phys. Chem. C, 2008, 112, 13563-13570.

12 T. A. Witten and L. M. Sander, Phys. Rev. Lett., 1981, 47, 1400-1403.

13 J. A. Corno, et al., J. Phys. Chem. C, 2008, 112, 5439-5446.

14 L. A. Peyser, et al., Science, 2001, 291, 103-106; G. De Cremer, et al., Angew. Chem., Int. Ed., 2008, 47, 2813-2816; G. De Cremer, B. F. Sels and J. Hotta, et al., Adv. Mater., 2010, 22, 957-960; G. De Cremer, E. Coutino-Gonzalez and M. B. J. Roeffaers, et al., ChemPhysChem, 2010, 11, 1627-1631; G. De Cremer, E. CoutiñoGonzalez and M. B. J. Roeffaers, et al., J. Am. Chem. Soc., 2009, 131, 3049-3056.

15 S. Mahajan, et al., J. Phys. Chem. C, 2010, 114, 7242-7250. 